\title{
Time-division multiplexing method for transmitting digital broadcasts over FTTH
}

\author{
Takeshi Kusakabe $^{1,2 a)}$, Takuya Kurakake ${ }^{3}$, Kimiyuki Oyamada ${ }^{4}$, \\ and Yoshihiro Fujita ${ }^{1}$ \\ ${ }^{1}$ Ehime University, Bunkyo 3, Matsuyama, Ehime 790-8507, Japan \\ ${ }^{2}$ NHK Matsuyama Broadcasting Station, Horinouchi 5, Matsuyama, Ehime \\ 790-8501, Japan \\ ${ }^{3}$ NHK Sendai Broadcasting Station, Nischikicho 1-11-1, Aoba-ku, Sendai, Miyagi \\ 980-8435, Japan \\ ${ }^{4}$ NHK Science \& Technology Research Laboratories, Kinuta 1-10-11, Setagaya-ku, \\ Tokyo, 157-8510, Japan \\ a)kusakabe.t-fi@nhk.or.jp
}

\begin{abstract}
We propose a novel time-division multiplexing method with a dedicated frame structure for transmitting digital broadcasts to homes over FTTH. By using experimental equipment, it was revealed that the proposed method can be expected to serve more subscribers than does the conventional frequency-division multiplexing method, and it achieves a low transmission latency and short period of program selection time. In this paper, we introduce and investigate the advantages and challenges for the future of the proposed method from the aspect of the requirements for broadcasting.
\end{abstract}

Keywords: digital broadcast, time-division multiplexing, FTTH

Classification: Terrestrial Wireless Communication/Broadcasting Technologies

\section{References}

[1] [Online] http://www.soumu.go.jp/english/index.html

[2] ITU-T Rec. G.987, 2012.

[3] IEEE 802.3av, 2010.

[4] ITU-T Rec. J.186, 2008.

[5] ITU-T Rec. J.185, 2002.

[6] S. Ikeda, T. Shitaba, H. Yoshinaga, and S. Aoyagi, "Video Signal Distribution System by Using FM Conversion Technique," IEICE Society Conference CS-5-4, 2008.

[7] ITU-R Rec. BT.2020, 2012.

[8] OITDA TP01/BW-2011, 2011.

[9] M. Nakamura, "Digital cable television system," J. Inst. Image Inform. TV. Engnr., vol. 65, no. 1, pp. 9-14, 2011.

[10] ITU-T Rec. Y.1901, 2009.

[11] K. Fujihara, Y. Kanayama, and T. Tsuboi, "A Realization and Performance Evaluation of a Failure-Resistant ATM Transport (FRAT) 
Method for High-Quality Video Transmission Services," IEICE Trans. Commun. (Japanese Edition), vol. J84-B, no. 2, pp. 157-166, 2001.

[12] ITU-T Rec. J.183, 2001.

[13] T. Kusakabe, T. Kurakake, N. Nakamura, and K. Oyamada, "Retransmission system for digital broadcasts using base-band multiplexing of MPEG-2 TS streams over FTTH," ITE Technical Report, vol. 30, no. 40, pp. 21-24, 2006.

[14] S. Ikeda, "Trend of optical video signal transmission systems," J. Inst. Image Inform. TV. Engnr, vol. 63, no. 4, pp. 437-443, 2009.

[15] Y. Hakamada, N. Nakamura, K. Oyamada, T. Kurakake, and T. Kusakabe, "An UHDTV cable television distribution in combinations of multiple 64 and 256 QAM channels," ICCE2013, pp. 490-491, 2013.

[16] ITU-T Rec. J.281, 2005.

[17] JCTEA TR-006, 1.0, 2005.

[18] T. Kusakabe, T. Kurakake, and K. Oyamada, "A Study of the Optical Distribution Costs of Multichannel Baseband Digital Broadcasts over a Fibre-To-The-Home Network," OECC2009, ThD4, 2009.

[19] NHK STRL annual report, p. 51, 2006.

[20] T. Kusakabe, T. Kurakake, and K. Oyamada, "Transmission delay and channel switching durations in a base-band transmission system of digital broadcasts," Proceedngs of the ITE winter annual convention, 8- 2, 2006.

[21] T. Kusakabe, T. Kurakake, and K. Oyamada, "A Multi-Channel ReModulator for In-House Coaxial Cable Transmission on Digital Broadcasting FTTH Systems," Proceedings of the ITE annual convention, 16 8, 2009.

\section{Introduction}

In recent years, fiber-to-the-home (FTTH) has come into full-scale use. Over $40 \%$ of cable television (CATV) operators have already shifted to FTTH [1]. As a communication access network, a passive double-star (PON) system, a form of FTTH, has been widely used. Now, a 10-Gbps class PON system $[2,3]$ is expected to be put into practical use.

To transmit broadcast signals over FTTH, the sub-carrier multiplexing (SCM) method [4] and FM conversion method [5, 6] (hereafter referred to as the FDM methods) have already been standardized. The FDM methods are applied to both analog (VSB-AM) and digital (OFDM, QAM) signals, the existing coaxial cable, and the conventional set top box (STB).

Moreover, ultra high definition television (UHDTV) was standardized [7]. We can expect an increase in the demand for a higher transmission bandwidth to homes with lower cost.

Considering the adaptation to such a new broadcast system, the progress on replacing metal cables with optical fiber even in houses [8], and the expiration of analog transmission by March 2015 [9] in Japan, it is now possible to apply time division multiplexing (TDM) of baseband digital broadcasting signals for distribution to homes, not FDM of RF signals. As examples of TDM being applied over an optical network, Internet Protocol television (IPTV) [10] was recently developed, and a TV trunk network based on asyn- 
chronous transfer mode (ATM) technology [11] has been put into practical use.

However, there are problems from the aspect of transmission efficiency and stability. IPTV has certain jitter and transmission latency problems caused by sharing bandwidth with communication signals. ATM has about a $9 \%(5 / 53)$ header overhead, which results in a low multiplexing efficiency, and has no forward error detection or correction for a payload.

In this paper, a novel TDM method is proposed that is suited for distributing digital broadcasts including UHDTV to homes with modified transport stream multiplexing frames (TSMFs) [12] by using the $1550-\mathrm{nm}$ wavelength in optical fiber exclusively for broadcasting. The purpose of this paper is to introduce and investigate the advantages and challenges for the future of the proposed method from the aspect of requirements for broadcasting, based on the basic optical transmission characteristics [13]. The proposed method is described in chapter 2 and is compared with conventional methods in chapter 3 . In chapters 4 and 5 , the required optical power, transmission latency, and program selection time are discussed.

\section{Proposing a TDM transmission method}
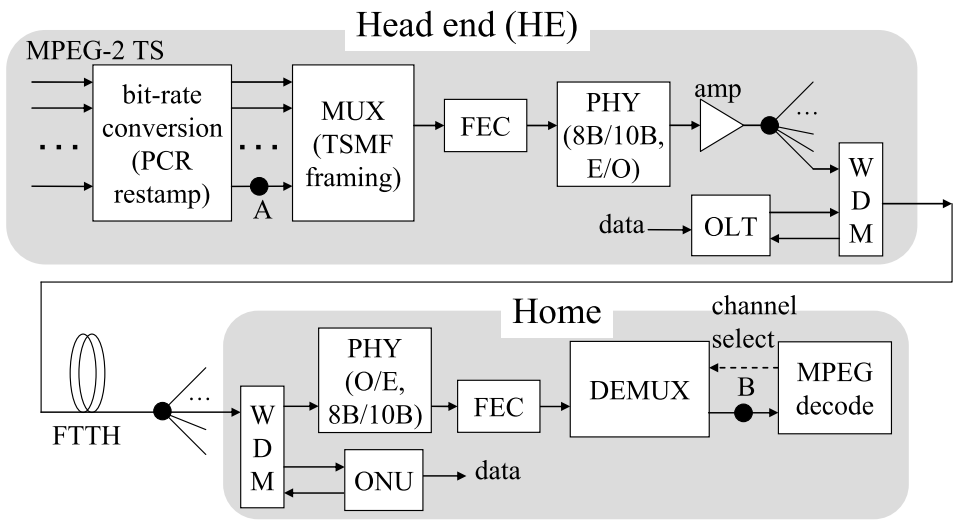

(a) Transmission system

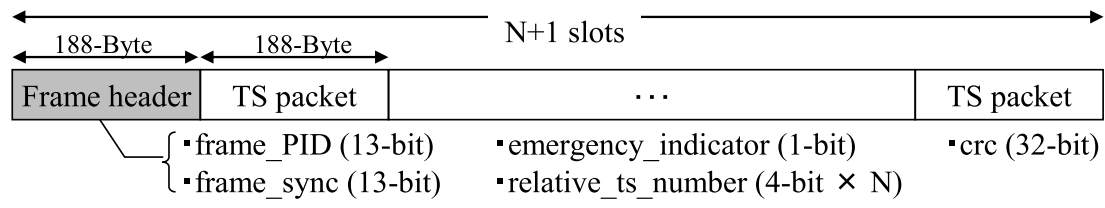

(b) Modified TSMF structure

Fig. 1. Proposed TDM method

Figure 1 (a) shows a proposed TDM transmission system. At the head end (HE), multiple programs of transport streams (TSs) are multiplexed into modified TSMFs and RS(204,188) error correcting codes are added as a FEC. Then, they are optically intensity-modulated. At subscribers' premises, an optically de-modulated baseband signal is de-multiplexed into TS packets after frame synchronization. A physical layer (PHY) block can be made to be compatible with communication standards that use the 1550-nm wavelength. 
Wavelength division multiplexing (WDM) with communication signals can be applied as well as the FDM methods.

Figure 1 (b) shows the structure of the modified TSMF. The number of data slots $(\mathrm{N})$ can be taken to be 222 at a maximum when the number of TSs is limited up to 15 , which results in a less than $1 \%$ header overhead.

Clock recovery and burst packet error caused by header error represents problems with applying the modified TSMF for the TDM transmission. As for the clock, it can be recovered by $8 \mathrm{~B} / 10 \mathrm{~B}$ or $64 \mathrm{~B} / 66 \mathrm{~B}$ encoding. As for the header error, HE converts the bit rate and restamps the PCR of the TS to match the allocated number of slots by using the CBR characteristic of each TS, which results in the static allocation of TS packets and keeps header information unchanged. If a header error is detected by a CRC check, the header information previously received can be used to de-multiplex.

\section{Comparison with conventional methods}

\subsection{Comparison with the FDM methods}

In this section, we compare the proposed method with the FDM methods from the aspect of the requirements as an optical transmission system [14], compatibility with conventional systems, and availability for a higher transmission bandwidth.

(1) Optical transmitter

The FDM methods require a high-linearity optical transmitter in order to suppress inter-carrier distortion. In contrast, the proposed method accepts a much lower linearity and an optical intensity modulation index of almost $100 \%$.

(2) Network

With the proposed method, an optical receiver accepts a signal at a lower power, the same as a communication signal, which allows for a greater number of branches in HE, as discussed in chapter 4, and enables line design to be moved to a common architecture with communication in the WDM section.

Whereas, the proposed method needs a wideband transmission line like an optical fiber instead of the conventional coaxial cable on subscribers' premises.

(3) STB

The conventional STB, which receives an RF signal, is not available for the proposed method. A dedicated STB with a baseband signal interface is required.

(4) Flexibility for TS bit rate

Signals that exceed the capacity of a single carrier must be divided into multiple streams [15], and the STB must prepare multiple tuners in the FDM methods. The proposed method requires no such partitioning, and high-bitrate streams can be transmitted without being divided into multiple low-bitrate streams. 


\subsection{Comparison with IPTV}

In this section, we compare the proposed method with IPTV from the aspect of the requirements for applying a wired IP-based network for broadcasting [16].

(1) Transmission and service

The proposed method achieves a higher multiplexing efficiency and stable, jitter-free transmission without packet loss, as mentioned in chapter 2, whereas IPTV needs a measure against jitter and packet loss. Further, the proposed method enables low transmission latency, as discussed in chapter 5, whereas IPTV has a certain latency due to buffers in routers and receivers.

(2) Multiplexing and program selection

The proposed method achieves a short period of program selection time even compared with a broadcast received directly, as discussed in chapter 5 , whereas an IPTV program is selected at a router in the network with a certain period of time.

(3) Network bandwidth

The proposed method essentially transmits all of the programs simultaneously and guarantees confidentiality of program selection.

(4) Coordination with other services and technologies

The proposed method can be independent of communication traffic with appropriate isolation of a WDM filter by using a wavelength for broadcasting.

\section{Required optical power}

Here, we discuss the optical power required at the receiver of the proposed method by using prototype equipment. The number of data slots $\mathrm{N}$ is set to be 48 , and the PHY of the method is set to comply with gigabit Ethernet.

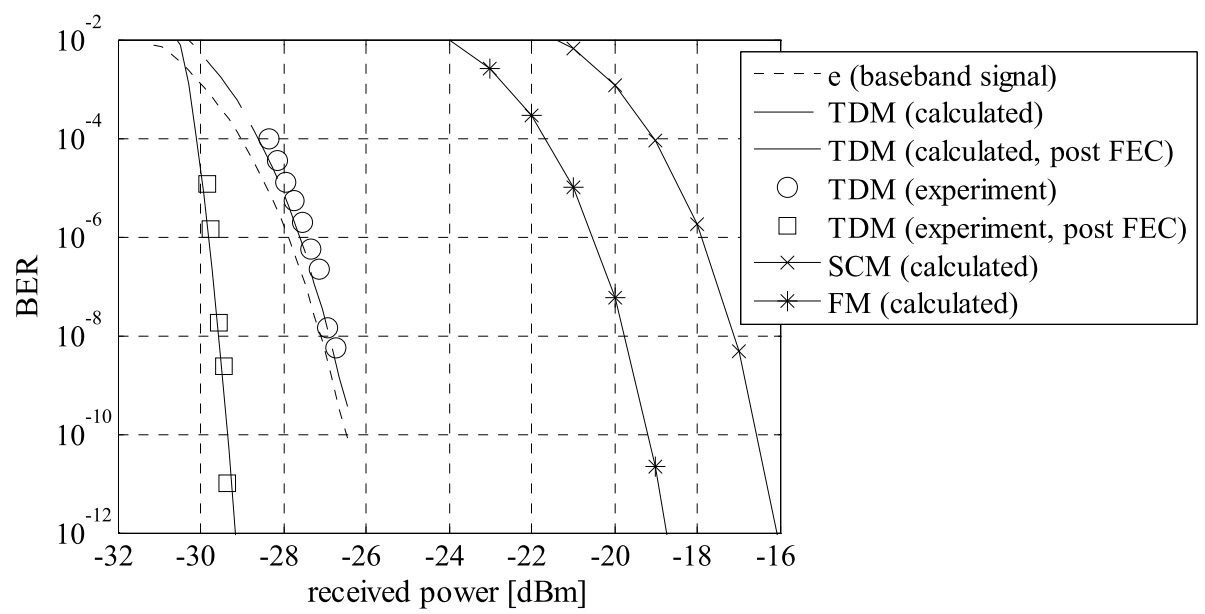

Fig. 2. BER vs. received power

The BERs of the received TS are shown in figure 2 with "experiment". For the BER of the baseband signal that was represented as e, the BER of the TS was calculated as 3.5 e, as shown in figure 2 with "calculated," because a 3.5 bit error occurred per 8 -bit blocks on average when the $8 \mathrm{~B} / 10 \mathrm{~B}$ 
decoder failed. The measured BER of the TS coincided approximately with the calculated BER. It confirmed that the influence of the modified TSMF framing on the required power was negligible.

The BERs of the FDM methods, calculated with the parameters in reference 17 are also shown with "SCM" and "FM," respectively. Both methods transmitted thirty 64-QAM signal, the total capacity of which was about 950 Mbps, including FEC.

The received power of the proposed method was 7-9-dB lower than that of the FDM methods. We can expect HE to serve more subscribers and drastically reduce the cost per subscriber up to $20-25 \%$ of that of the SCM and FM conversion methods [18] by this result.

\section{Transmission latency and program selection time}

In this chapter, we discuss the transmission latency and program selection time of the proposed method. Experimental results by using the prototype equipment $[19,20]$ are shown in the following.

The transmission latency of the proposed method, which corresponds to the section from A to B in figure 1 (a), was measured to be about $3 \mathrm{~ms}$, due mainly to the framing process (about $2.6 \mathrm{~ms}$ ), FEC, and $8 \mathrm{~B} / 10 \mathrm{~B}$ encoding (about $0.4 \mathrm{~ms}$ ). The latency was negligibly small from a practical viewpoint.

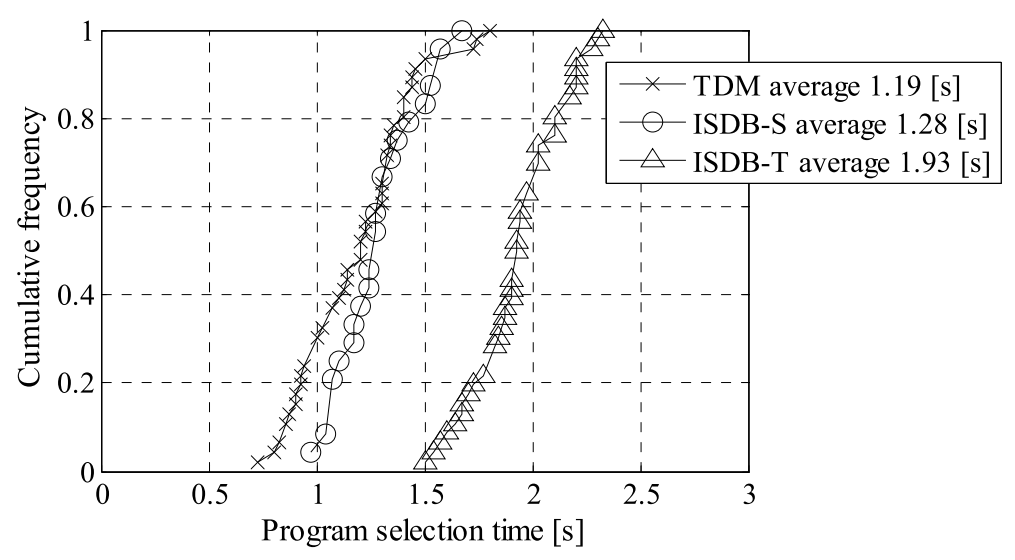

Fig. 3. Program selection time

Figure 3 shows the program selection time of the proposed method and the conventional direct-reception tuners, e.g., ISDB-T and ISDB-S, as a cumulative frequency.

The time of the proposed method became shorter on average because the method has no processes such as frame synchronization (231-463 ms for ISDB-T, $1.4-2.8 \mathrm{~ms}$ for ISDB-S) and time de-interleaving (218 ms, $11 \mathrm{~ms}$ at a maximum for ISDB-T and ISDB-S, respectively) at every program selection. The receiver of the proposed method does not need select-by-select frame synchronization if once synchronized after the receiver is turned on. 


\section{Conclusion}

We proposed a novel TDM method for digital broadcasting to homes over FTTH and compared it with FDM methods and IPTV from the aspect of requirements for broadcasting. The experimental results revealed that the proposed method can serve more subscribers compared with the FDM methods, and it achieves a low transmission latency and shorter program selection time. The proposed method is useful under the particular conditions that consideration of compatibility with the conventional CATV system, which transmits RF signals, is not needed and that there is an optical fiber on subscribers' premises.

To expand the availability of the proposed method for the future, a measure that enables simple migration from the conventional CATV system to the proposed system and an additional method [21] compatible with coaxial cable and conventional STB should be considered. 